\title{
Spatial cognition and memory: a reversible lesion with lidocaine into the anteromedial/posterior parietal cortex (AM/PPC) affects differently working and long-term memory on two foraging tasks
}

\author{
PABLO ESPINA-MARCHANT ${ }^{1}$, TERESA PINTO-HAMUY ${ }^{1}$, DIEGO BUSTAMANTE ${ }^{2}$, \\ PAOLA MORALES ${ }^{2}$, LUIS ROBLES ${ }^{1}$ and MARIO HERRERA-MARSCHITZ ${ }^{2 C A}$
}

\footnotetext{
${ }^{1}$ Programmes of Physiology \& Biophysics, and ${ }^{2}$ Molecular \& Clinical Pharmacology, ICBM, Medical Faculty, University of Chile, Santiago, Chile.
}

\begin{abstract}
Place memory is relevant for exploration and forage behaviour. When food supply is dispersed, a win-shift has advantage over a win-stay strategy. In the Olton Octagonal Maze, the rat follows a win-shift strategy using working memory. However, in the Olton $4 \mathrm{x} 4$ version, the rat follows a win-stay strategy, using both working and long-term memories. It has been suggested that the neocortex is required for the resolution of tasks demanding long-term, but not for that demanding working memory alone. The role of anteromedial/posterior parietal cortex (AM/PPC) was investigated here, using a reversible lesion induced by intracerebral lidocaine infusion. Long-Evans rats were implanted with guide cannulae into the AM/PPC and trained in an Olton 4x4 maze, counting working and long-term memory errors after a delay. Then, the animals were infused with lidocaine or saline during the delay phase and tested for three days. Another series of animals, treated as before, was tested in an Olton Octagonal Maze and subjected to the same injection schedule. In the Olton $4 x 4$ Maze, lidocaine produced a significant increase in working and long-term memory errors, compared to saline and post-lidocaine conditions. In contrast, in the Olton Octagonal Maze, lidocaine did not induce any effect on working memory errors. Thus, AM/PPC is required when both working with previous information and long-term memories are needed, but not when only working memory is required, as it happens under ethological conditions. Whenever food supply is dispersed, a win-shift strategy is preferable.
\end{abstract}

Key terms: Anteromedial/posterior parietal cortex, hippocampus, lidocaine, long-term memory, working memory, intracerebral infusion, rat.

\section{INTRODUCTION}

Spatial cognition and memory is today an active field of research. The ability to efficiently locate and retrieve food is an essential survival strategy for rodents. The pioneer works of Olton [17, 18], who developed the Octagonal Maze, provided evidence that rodents use a win-shift strategy for locating and retrieving food, abandoning an initial site of food discovery to search for another site. The win-shift is more appropriate than the win-stay strategy, in particular under conditions when food supply is dispersed, which happens in the natural environment of Long Evans rats. Olton and Samuelson [17] demonstrated that working memory is involved in the solution of the task when food is dispersed. Working memory implies the temporary activation of a memory network, as needed for the execution of successive acts in a temporal structure of behaviour [8]; and in this case, for recovering the food from different places using a win-shift strategy. From a neurological point of view, O'Keefe and Dostrovsky [15] and O'Keefe and Nadel [16] proposed that the so called "place cell" is in the hippocampus, mainly in $\mathrm{CA} 1$ region, meaning that in that region 
there is a group of cells showing locationspecific firing [15]. That idea has generated enormous amount of research. Also, the connections of the hippocampus and its adjacent structures, like enthorrinal cortex, are involved for conveying a wide variety of sensorial cues [34, 35].

Using a delayed spatial win-shift task, that is, a modified Olton Octagonal maze with three phases, i.e. training, delay and test (Olton 4x4 maze with delay) for studying spatial memory, Floresco et al. [7] demonstrated that transient inactivation of the involved neurocircuitries with lidocaine differentiated between two neurological pathways related to foraging tasks in Long Evans rats, whether previous information is or not required. When previous information is required, that information is stored as long-term memory, and the rat will use it to recover the food more efficiently, using a win-stay strategy for location of the food storage [7]. When previous information is not required, the animal will adopt a winshift strategy, using working memory only.

Long-term memory, as evaluated by the Olton $4 \times 4$ maze with delay, comprises a neurocircuitry involving at least three structures: (i) CA1/subiculum, (ii) prelimbic cortex (PL), and (iii) nucleus accumbens. When previous information is not required, implying working memory only (evaluated with the Olton Octagonal maze without delay), the neurocircuitry comprises only two structures: (i) CA1/subiculum and (ii) accumbens. Hence, the inactivation of nucleus accumbens with lidocaine induces a deficit in both working and long-term memory, the rats failing in the Olton $4 \times 4$ maze with delay, as well as in the Olton Octagonal maze without delay [7].

Pinto et al. [23] investigated the role of the anteromedial/posterior parietal cortex (AM/ PPC) in bimodal visuo-spatial discrimination, training rats in a modified Sutherland apparatus, with a gradual procedure (fading), consisting in a retractable jumping platform and a tunnel lined with two different roughness papers, associated with either a white or a black landing door. In that study, rats with an ibotenate lesion into the AM/PPC failed the test, suggesting that the AM/PPC area is involved in the integration of visual and somatic stimuli [23]. The lesioned rats also failed in learning a visuospatial discrimination task assessed in a Skinner box [27]. The role of the AM/PPC was then evaluated in a Morris water maze, finding that lesioned rats showed a significant increment in latency to reach the platform [6].

Furthermore, an ibotenate lesion of the rostral part of Oc2M (a region of the AM/ PPC complex) produced a retention deficit in the Lashley III maze [22]. The deficit was positively correlated with the size of the lesion, thus indicating that the AM/PPC complex is involved in storing spatial information [22]. The hippocampus-parietal cortex interaction is important for handling visuo-spatial information [20, 24, 25], while the parietal neocortex is involved in a more abstract spatial processing [24], when cognitive functions are required $[1,31]$. These studies suggested that AM/PPC is relevant for long-term memory, integrating different sensory modalities and egocentric cues for constructing an allocentric representation of the environment.

Considering the hypothesis that AM/ PPC is involved in spatial memory when allocentric behaviour is required for the resolution of long-term memory tasks [6, 22 , see 8,30$]$, we postulated that, in addition to hippocampus, the AM/PPC complex is also involved when both working and long-term memory are required. Thus, we investigated the effect of a transient lesion of the AM/PPC with an intracerebral lidocaine injection on learning behaviour assessed in an Olton $4 \times 4$ with delay, and in an Olton Octagonal maze without delay, requiring or not previous information.

The present study has been fulfilled to honour the memory of Prof. Dr. Teresa Pinto-Hamuy, a pioneering and inspiring teacher for a long generation of scientists interested on behavioural neuroscience.

\section{MATERIALS AND METHODS}

\section{Subjects}

Long Evans rats, approximately 3 months old (200 g), were stereotaxically implanted 
with guide cannulae, and food-deprived, preserving free access to water, for three days until a decrease of $10 \%$ of their original body weight was achieved. All animals were first allowed to explore in $a$ classical Olton Octagonal Maze without delay (Fig. 1A). Then, a group of rats was used for Experiment I in an Olton 4x4 Maze with delay (Fig. 1B, C). Thereafter, that group was split into two subgroups, depending on whether the rats received an intracerebral infusion of lidocaine or saline. Another group of rats was used for Experiment II, performed in the Classical Olton Octagonal Maze without delay.

When not in the experimentation room, the rats were individually housed in home cages, under a 12/12 h inverted light/dark cycle (light off at 08: $00 \mathrm{hr}$ ). The experiments were evaluated by a local committee for the ethics of experiments with animals (TP-H, DID S9608/2; DID SOO-01/2; MH-M, Protocol CBA\# 0136, FMUCH), endorsing the principles of laboratory animal care $\left(\mathrm{NIH}\right.$; $\mathrm{N}^{\mathrm{o}} 86-23$; revised 1985).

\section{Surgery}

The rats were administered chloral hydrate $(0.5 \mathrm{ml}, 7 \%$ solution; i.p.), and placed in a Kopf stereotaxic apparatus. Gaseous anaesthesia was further kept with a mixture of air and isoflurane $(1.5 \%)$ by a mask fitting to the nose of the animal. A puff of lidocaine (Xylocain $10 \mathrm{mg} / \mathrm{ml}$; AstraZeneca, Södertälje, Sweden) was applied on the skin to be cut for exposing the skull. Two holes ( $2 \mathrm{~mm}$ diameter) were opened through the skull with a trephine drill to expose the dura, allowing the bilateral implantation of guide cannulae (CMA 7 model by CMA/Microdialysis Solna, Sweden) into the peristriate AM/PPC visual area, using the following coordinates according with the atlas of Paxinos and Watson [21]: AP-4.2 mm; ML $\pm 3.4 \mathrm{~mm}$ and DV $0.5 \mathrm{~mm}$. The guide cannulae were fixed with dental acrylate anchored to two screws fixed to the skull. At the end of the experiments the correct site of the cannulae implantation was verified at low magnification for each animal, excluding any case where the implantation was not in the targeted area.

\section{Intracerebral Infusion Procedure}

Two to three weeks after surgery, the stainless steel tubing sealing the guide cannulae was removed to allow the insertion of the injection probes of $0.3 \mathrm{~mm}$ of diameter into the target region for the intracerebral infusion of $1 \mu \mathrm{L}$ of a $2 \%$ lidocaine (Lidocaine hydrochloride, Sanderson Lab. S.A., Santiago, Chile) or saline solution by a microinjection pump (CMA/100; CMA/Microdialysis Solna, Sweden), set at a delivery rate of $0.5 \mu \mathrm{L} /$ min. The injection probes remained in the guide cannulae to allow drug diffusion for one additional min before the rats were replaced in their home cage and/or into the testing apparatus.

\section{Testing Apparatus}

The experimentation room was equipped with artificial lighting and a variety of visual signals on the walls, which were maintained during all the trials. The Olton Octagonal Maze was placed at a height of $92 \mathrm{~cm}$ above the floor. An octagonal platform, $34 \mathrm{~cm}$ in diameter, was placed in the centre of the maze, connecting 8 arms of equal length $(71 \mathrm{~cm}$ long $x 7.5$ $\mathrm{cm}$ wide) distributed radially. The bait was placed in a circular receptacle at the far end of each arm, hidden from the view of the rat.

A schematic representation of the experimental setup is in Fig. 1, modified from Bustos et al. [2]. Before Experiment I, there was a three days habituation period, letting the rats explore the arms of the Olton Octagonal Maze loaded with grains of puffed rice (Fig. 1A). The same maze was modified as an Olton $4 x 4$ with delay, but with a $4 \times 4$ modification, referring to a randomised blocking of the lanes during the first training phase (Fig. 1B). For the test phase, the bait was placed on the previously blocked lanes (Fig. 1C). For Experiment II, the apparatus was re-organised as a classic Olton Octagonal Maze, with the 8 arms, opened and baited. 

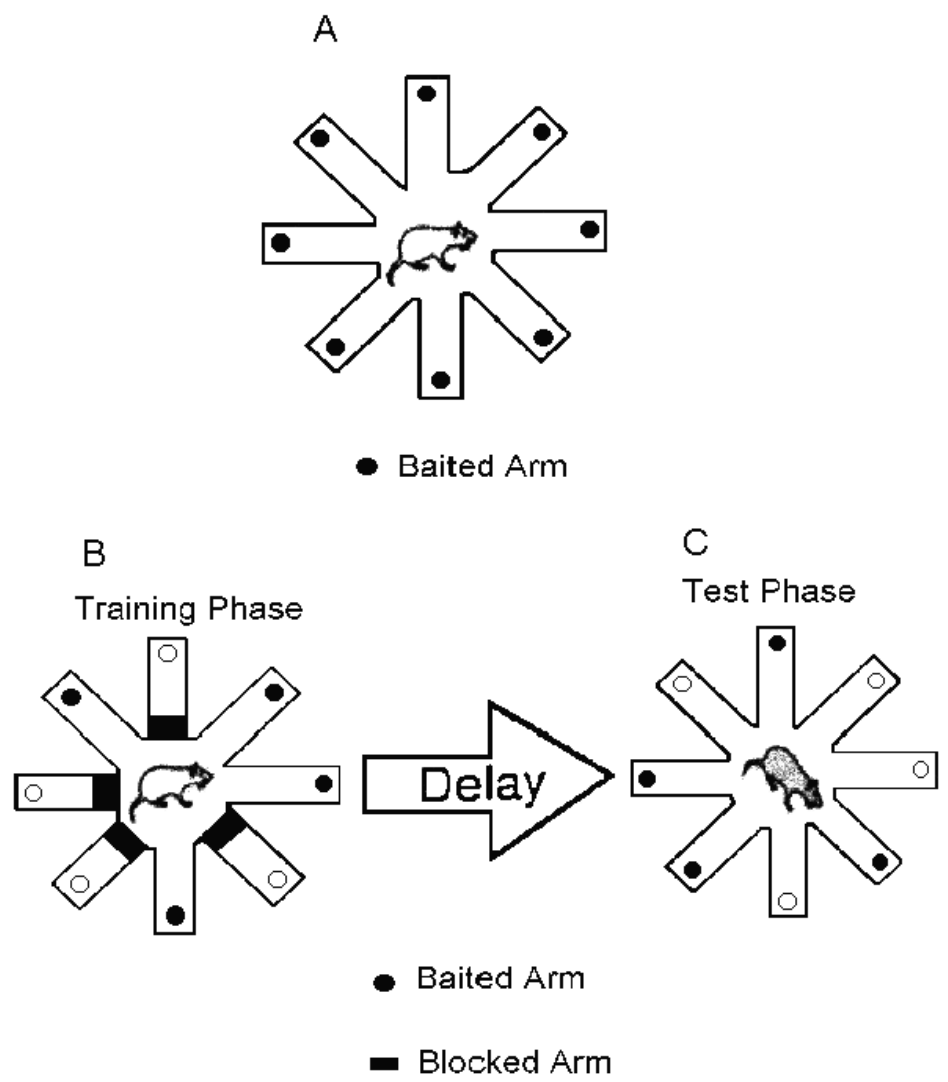

Figure 1: A schematic diagram of the Olton Octagonal Maze is shown in A, with the 8 arms opened and baited. The same maze was modified as an Olton $4 x 4$ with delay, with a randomised blocking of the lanes during the first training phase (B). For the test phase, the bait was placed on the previously blocked lanes, and all arms were opened (C). The large arrow indicates the delay phase, taking place in the home cage. The figure is a modified version of a diagram reported by Bustos et al. [2].

\section{Design and Procedure}

There was, first, a habituation period, consisting of exposing the rats to the Olton Octagonal Maze (with the 8 arms, opened, and baited) for $10 \mathrm{~min}$ along three days (Fig. 1A). Thereafter the animals were used for Experiment I or II. For Experiment I, the rats were exposed to the Olton $4 x 4$ Maze with delay. For Experiment II, the rats were exposed to the Olton Octagonal Maze without delay. All sessions were properly video-taped.

\section{Experiment I: Olton $4 x 4$ with delay}

The animals were observed before and following a delay, with or without intracerebral infusion. For each experimental session there was: (i) a training, (ii) a delay, and (iii) a test phase.

(i) Training phase. During this phase only 4 arms of the maze were loaded with the bait, while the other 4 arms, selected at random, were blocked (Fig. 1B). The rat was placed in the central platform and required to enter into any of the 4 nonblocked arms to retrieve the bait for a maximum period of $10 \mathrm{~min}$.

(ii) Delay phase. It refers to the period when the rat was returned to its home cage, staying there for $8 \mathrm{~min}$ or $25 \mathrm{~min}$.

(iii) Test phase. During this phase the animal was re-placed in the Olton $4 \times 4$ maze, with all arms opened (Fig. 1C), but baits only placed in the arms that were previously 
blocked during the training phase. The rat was expected to enter the arms that were blocked during the training phase and to retrieve the bait placed there. The four nonblocked arms during the training phase had no bait during the test phase.

During the test phase, two types of errors were evaluated: (i) working memory error, assigned when the rat re-entered into an already visited arm during the same test phase, i.e. when the rat entered into an arm without bait, because it had already retrieved the bait during the current test phase. (ii) Long-term memory error, assigned when the rat entered into an already visited arm during the training phase, which had no bait during the test phase by design. The learning criterion was: no more than one working or one long-term memory error during the test phase, for each animal along three consecutive days. Before the intracerebral infusion, all animals had already completed 15 sessions, reaching the learning criterion.

The intracerebral infusion schedule comprised three further consecutive daily sessions, i.e. (i) saline; (ii) lidocaine or saline, or (iii) post-lidocaine or post-saline sessions.

(i) Saline. After carrying out the training phase, the animals received a saline infusion during the delay phase in their home cage (see intracerebral infusion procedure). After the infusion the cannulae were drawn out, the rats were transported to the experimentation room to complete the delay phase. Thereafter, the rats were replaced in the Olton $4 \times 4$ Maze for carrying out the test phase, recording working and long-term memory errors.

(ii) Lidocaine or Saline. The following day, all rats began the training phase, but split into two subgroups, injected with lidocaine or saline during the delay phase. After that phase, as above, the rats were replaced into the Olton $4 \times 4$ Maze, carrying out the test phase, recording working and long-term memory errors.

(iii) Post-lidocaine or post-saline. At this stage, no infusion was made during the delay phase, but working and long-term memory errors were recorded during the test phase.
Two final sessions were performed with a 25 min delay, to confirm the long-term memory effect, but repeating the intracerebral infusion schedule indicated above, with a saline, and lidocaine and/or saline sessions.

Experiment II: Olton Octagonal Maze without delay.

A series of rats was trained without delay, in the classical Olton Octagonal Maze, with the 8 arms open and loaded with the bait. The rat was placed in the central platform of the maze and required to forage the bait for no more than 10 minutes. The rat was expected to only enter the non visited arms. During this unique phase, a working memory error was recorded every time the rat re-entered into an already visited arm, therefore, only working memory errors were recorded. Once the animals reached the learning criterion (average of one working memory error for each animal along three consecutive days), the intracerebral infusion schedule, saline, lidocaine and post-lidocaine was carried out.

The intracerebral infusion schedule was carried out along three consecutive daily sessions, i.e. (i) saline; (ii) lidocaine, and (iii) post-lidocaine.

(i) Saline. During this session, the rats received a saline infusion in their home cage, before being placed into the maze. After the infusion the cannulae were drawn out, and the rats were transported to the experimentation room. Thereafter, the rats were placed into the Olton Octagonal Maze for carrying out the test phase, recording working memory errors.

(ii) Lidocaine. The rats received the lidocaine solution, and working memory errors were recorded. Intracerebral infusion of saline was not performed, because its lack of effect was reported previously [6].

(iii) Post-lidocaine. During this session, the animals were re-placed into the testing apparatus without receiving any infusion, but recording working memory errors.

\section{Statistics}

The results were analysed with the statistical software STATA 8.1. The results are 
expressed as the means \pm SEM. The Wilcoxon test was used for paired comparisons of error scores between sessions. The nonparametric U Mann-Whitney test was used for non-paired comparisons; i.e. lidocaine versus saline, and/or post-lidocaine versus post-saline, when the error scores were analyzed at the same session. A level of $\mathrm{p}<0.05$ was considered for statistically significant differences.

\section{RESULTS}

\section{Experiment I Olton $4 \times 4$ with delay}

Working memory with delay (Table 1)

Table 1 shows the effect of saline or lidocaine on working memory errors following 8 or 25 min delay. A significant increase in the amount of errors was observed following intracerebral lidocaine infusion. The length of the delay phase did not make any difference on the magnitude of the error increase. No effect was observed following a single or repeated saline infusion. The effect of lidocaine was transient, because no effect on working memory errors was observed when the same animal was tested $24 \mathrm{~h}$ after the drug, following 8 min delay.

\section{Long-term memory with delay (Table 2)}

Table 2 shows the effect of saline or lidocaine on long-term memory errors following 8 or 25 min delay. As for working memory, a significant increase in the amount of errors was observed following intracerebral lidocaine infusion. The length of the delay phase did not make any difference on the recorded errors. No effect was observed following a single or repeated saline infusion. The effect of lidocaine was transient, because no effect on long-term memory errors was observed when the same animal was tested $24 \mathrm{~h}$ after the drug, following 8 min delay.

\section{Experiment II. Olton Octagonal maze without delay (Table 3)}

A group of rats was trained in the Classical Olton Octagonal Maze, and tested under (i) saline, (ii) lidocaine and (iii) post-lidocaine conditions for evaluating working memory without delay. No significant effect on working memory was observed immediately or $24 \mathrm{~h}$ after lidocaine treatment.

\section{DISCUSSION}

Using a reversible lesion model induced by intracerebral lidocaine, Seamans and Phillips [32] suggested a neurocircuitry for foraging behaviour, comprising the nucleus accumbens and hippocampus. Floresco et al. [7] further demonstrated that the CA1/ subiculum, prelimbic, and accumbens regions are involved in a foraging task with long delay, suggesting that memory traces have to be stored in the prefrontal neocortex of the rat, where a prospective sequence for cognition and motor planning is organized [7]. The prelimbic region of the prefrontal neocortex would play a role in the retrieval of spatial information required to predict the probable location of food during the test phase of an Olton 4x4 maze.

The present results show that both working and long-term memory errors, assessed with the Olton $4 \times 4$ with delay maze, were significantly increased following a lidocaine infusion into the AM/PPC, compared to saline, post-lidocaine or post-saline conditions, suggesting that that neuronal complex should also play a role in the neurocircuitry required for retrieval of spatial information.

It has been demonstrated [18] that the neocortex is not required for solving a task like that requested by the classical Olton Octagonal maze, where a rat uses working memory only. In agreement, we here show that an intracerebral lidocaine infusion into the AM/PPC cortex did not change the error scores measured in that maze, but, as shown above, the working memory errors increased in the Olton $4 \times 4$ with delay. Thus, when solving a task that does not require previous information, the cortical connections are not utilized. But, when the resolution of a task requires previous information, as seen in the Olton 4x4 with delay, the neurocircuitry becomes corticalized [2,24,25], involving prefrontal cortex, as suggested by Floresco et al. [7], but also the AM/PPC, as show here. 
TABLE $1 \mathrm{~A}, \mathrm{~B}$

Experiment I: working memory with delay

Number of working memory errors in Olton 4x4 maze following 8 (A) and 25 (B) min delay. Errors, as defined in the method section, are expressed as the means $\pm \mathrm{SEM} ;{ }^{\mathrm{a}} \mathrm{p}<0.05$ (underlined, bold), versus errors observed after saline I; ${ }^{b} \mathrm{p}<0.05$ (underlined, bold), versus errors observed after saline II; n.d., not done.

\begin{tabular}{|c|c|c|c|}
\hline A. Working memory (8 min delay) & Saline (I) & Saline (II)/Lidocaine & Post \\
\hline Saline $(n=4)$ & $0.8 \pm 0.3$ & $0.5 \pm 0.3$ & $0.3 \pm 0.3$ \\
\hline Lidocaine $(n=6)$ & $0.7 \pm 0.3$ & $\underline{3.8} \pm \underline{0.2} \underline{a, b}$ & $0.7 \pm 0.2$ \\
\hline \multicolumn{4}{|l|}{ B. Working memory (25 min delay) } \\
\hline Saline $(n=4)$ & $0.1 \pm 0.1$ & $0.3 \pm 0.2$ & n.d. \\
\hline Lidocaine $(n=6)$ & $0.1 \pm 0.1$ & $\underline{4.7} \pm \underline{0.4} \underline{\mathrm{a}, \mathrm{b}}$ & n.d. \\
\hline
\end{tabular}

TABLE 2A, B

Experiment I: long-term memory with delay

Number of long-term memory errors in Olton 4x4 maze following 8 (A) and 25 (B) min delay. Errors, as defined in the method section, are expressed as the means \pm SEM; ${ }^{\mathrm{a}} \mathrm{p}<0.05$ (underlined, bold), versus errors observed after saline I; ${ }^{b} \mathrm{p}<0.05$ (underlined, bold), versus errors observed after saline II; n.d., not done.

\begin{tabular}{|c|c|c|c|}
\hline A. Long-term memory (8 min delay) & Saline (I) & Saline (II)/Lidocaine & Post \\
\hline Saline $(n=4)$ & $1.8 \pm 0.5$ & $1.5 \pm 0.3$ & $2.0 \pm 0.4$ \\
\hline Lidocaine $(n=6)$ & $1.7 \pm 0.2$ & $\underline{4.2} \pm \underline{0.3} \underline{a}^{\mathrm{a}, \mathrm{b}}$ & $1.8 \pm 0.3$ \\
\hline \multicolumn{4}{|l|}{ B. Long-term memory ( 25 min delay) } \\
\hline Saline $(n=4)$ & $1.9 \pm 0.2$ & $0.5 \pm 0.3$ & n.d. \\
\hline Lidocaine $(n=6)$ & $2.0 \pm 0.4$ & $\underline{5.0} \pm \underline{0.5} \underline{a, b}^{a, b}$ & n.d. \\
\hline
\end{tabular}

TABLE 3

Experiment II: working memory without delay

Number of working memory errors in Olton Octagonal Maze. Errors, as defined in the method section, are expressed as the means \pm SEM.

\begin{tabular}{llll}
\hline Working memory) & Saline) & Lidocaine & Post \\
\hline Lidocaine $(\mathrm{n}=4)$ & $3.0 \pm 0.4$ & $2.5 \pm 0.3$ & $3.0 \pm 0.4$
\end{tabular}


Probable neural neurocircuitries underlying spatial memory comprise reciprocal connections of $\mathrm{AM} / \mathrm{PPC}$ to the retrosplenial cortex [28, 33, 34], and between the retrosplenial cortex and the hippocampal formation [35]. The hippocampus is a central structure for integrating multi-modal signals, allowing the configuration of a cognitive spatial map $[11,15,16]$. It is involved in episodic and spatial memory [26], containing cells that code for spatial location, the so called 'place cells' [11, 15, 16]. The present results suggest that the AM/PPC complex is critical for long-term, but also for working memory requiring previous spatial information, probably conveyed by hippocampus 'place cells' [22]. Indeed, these cells have been attributed with the capacity to integrate propioceptive, vestibular, and visual cues in rats $[11,12$, 14, 19].

Also, there is a connection between parietal and prefrontal cortex in rats [10, 13], suggesting that $\mathrm{AM} / \mathrm{PPC}$ is the integrator for stored spatial and perceptual information. This information has to be sent forward to the prefrontal cortex, where a decision is initiated. A subpopulation of neurons in the rostral part of the Oc2M (within the AM/PPC complex), which codes for head direction in absence of any visual cues, can provide a neural substrate for spatial memory in the AM/PPC complex [3]. These cells code mnemonically for directional movement and spatial representations on the basis of egocentric, visual or acoustic cues [4, 5, 14, 29, 30]. However, when Long Evans rats are faced with a new situation, not requiring previous information about food location, the animals change their strategy, using a neurocircuitry based on hippocampus and accumbens $[7,24,29,30,31]$. This is supported by the present results, since the AM/PPC lesion did not affect working memory, when previous information was not required for the resolution of the task.

The retrieval of old, and the formation of new perceptual memories, seem to imply the activation of a vast neuronal network of the posterior neocortex $[1,8,9]$. If an evoked memory is associated with an action, then, the activated network extends to the frontal lobe. The need to hold memory for prospective actions leads to the recruitment of prefrontal neurocircuitries. In addition, these networks send tonic inputs to the posterior neocortex, maintaining the perceptual network active until the action is completed [8,9].

In conclusion, the present results suggest that $\mathrm{AM} / \mathrm{PPC}$ is critical for long-term memory, and for working memory requiring previous information. However, the AM/ PPC is not required when the rat uses working memory only, as happens under ethological conditions. Whenever food supply is dispersed, a win-shift strategy is preferable.

\section{ACKNOWLEDGEMENTS}

The work was supported by DID (S9608/2; SOO-01/2) and FONDECYT (1030521). The excellent technical contribution of $\mathrm{Mr}$. Aníbal Martínez is warmly appreciated. The statistic advice by Dr. Waldo Aranda is acknowledged.

\section{REFERENCES}

1 ANDERSEN RA, SNYDER LH, BRADLEY DC, XING J (1997) Multimodal representation of space in the posterior parietal cortex and its use in planning movements. Annu Rev Neurosci, 20: 303-330

2 BUStOs G, BASOALTO E, PINTO-HAMUY T (2003) Spatial memory in Long Evans and Rattus Norvegicus rats. Biol Res, 36: 193-199

3 CHEN LL, LIN L-H, BARNES CA, MCNAUGHTON BL (1994) Head-direction cells in the rat posterior cortex. II. Contributions of visual and ideothetic information to the directional firing. Exp Brain Res, 101: 24-34

4 CHEN LL, LIN L-H, GREEN EJ, BARNES CA, MCNAUGHTON BL (1994) Head-direction cells in the rat posterior cortex. I. Anatomical distribution and behavioral modulation. Exp Brain Res, 101: 8-23

5 CHEN LL, NAKAMURA K (1998) Head-centered representation and spatial memory in rat posterior parietal cortex. Psychobiol, 26: 119-127

6 ESPINOZA S, PINTO-HAMUY T, PASSIG C, CARREÑO F, MARCHANT F, URZUA C (1999) Deficit in the Water-Maze after lesions in the anteromedial extrastriate cortex in rats. Physiol Behav, 66: 493-496

7 FLORESCO S, SEAMANS J, PHILLIPS A (1997) Selective roles for Hippocampal, Prefrontal cortical and Ventral Striate Circuits in Radial-Arm Maze Tasks with or without delay. J Neurosci, 17 (5): 1880-1890.

8 FUSTER JM (2000) Cortical dynamics of memory. Int J Psychophysiol, 35: 155-164 
9 FUSTER JM (2000) Prefrontal neurons in networks of executive memory. Brain Res Bull, 52: 331-336

$10 \mathrm{HOH}$ TE, KOLB B, EPPEL A, VANDERWOLF CH, CAIN DP (2003) Role of the neocortex in the water maze task in the rat: a detailed behavioral and GolgiCox analysis. Behav Brain Res 138: 81-94

11 MCNAUGHTON BL, BARNES CA, GERRARD JL, GOTHARD K, JUNG MW, KNIERIM JJ, KUDRIMOTI H, QIN Y, SKAGGS WE, SUSTER M, WEAVER KL (1996) Deciphering the hippocampal polyglot: the hippocampus as a path integration system. J Exp Biol, 199: 173-185

12 MCNAUGHTON BL, LEONARD B, CHEN L (1989) Cortical-hippocampal interactions and cognitive mapping: A hypothesis based on reintegration of the parietal and inferotemporal pathways for visual processing. Psychobiol, 17: 230-235

13 MITCHELL BD, CAULLER LJ (2001) Corticocortical and thalamocortical projections to layer I of the frontal neocortex in rats. Brain Res, 921: 68-77

14 NAKAMURA K (1999) Auditory spatial discriminatory and mnemonic neurons in rat posterior parietal cortex. J Neurophysiol, 82: 2503-2517

15 O'KEEFE J, DOSTROVSKY J (1971) The hippocampus as a spatial map. Preliminary evidence from unit activity in the freely moving rat. Brain Res, 34: 171-175

16 O'KEEFE J, NADEL L (1979) Precis of O'Keefe and Nadel's, the hippocampus as a cognitive map. Behav and Brain Sci, 2: 97-116

17 OLTON D, SAMUELSON R (1976) Remembrance of placed passed: Spatial memory in rats. J Exp Psychol: Animal Behav Processes, 2: 97-116

18 OLTON D, COLLISON C, WERZ M (1977) Spatial memory and radial maze performance of rats. Learning and Motivation, 8: 289-314

19 O'REILLY RC, RUDY JW (2001) Conjunctive representations in learning and memory: principles of cortical and hippocampal function. Psychol Rev, 108: 311-345

20 PARRON C, POUCET B, SAVE E (2001) Reevaluation of the spatial memory deficits induced by hippocampal short lasting inactivation reveals the need for cortical co-operation. Behav Brain Res, 127: 71-79

21 PAXINOS G, WATSON CH (1986) The Rat Brain in Stereotaxics Coordinates. London, Academic Press

22 PINTO-HAMUY T, MONTERO VM, TORREALBA F (2004) Neurotoxic lesion of anteromedial/posterior parietal cortex disrupts spatial maze memory in blind rats. Behav Brain Res, 153: 465-470

23 PINTO-HAMUY T, OLAVARRÍA J, GUIC-ROBLES E, MORGUES M, NASSAL O, PETIT D (1987) Rats with lesions in anteromedial extraestriate cortex fail to learn a visuosomatic conditional response. Behav Brain Res, 25: 221-231

24 POUCET B (1993) Spatial cognitive maps in animals: new hypotheses on their structure and neural mechanisms. Psychol Rev, 100: 163-182

25 POUCET B, BENHAMOU S (1997) The neuropsychology of spatial cognition in the rat. Crit Rev Neurobiol, 11: 101-120

26 ROLLS ET (2000) Memory systems in the brain. Annu Rev Psychol, 51: 599-630

27 SANCHEZ RF, MONTERO VM, ESPINOZA SG, DIAZ E, CANITROT M, PINTO-HAMUY T (1997) Visuospatial discrimination deficit in rats after ibotenate lesions in anteromedial visual cortex. Physiol Behav, 62: 989-994

28 SANDERSON KJ, DREHER B, GAYER N (1991) Prosencephalic connections of striate and extrastriate areas of rat visual cortex. Exp Brain Res, 85: 324-334

29 SAVE E, CRESSANT A, THINUS-BLANC C, POUCET B (1998) Spatial firing of hippocampal place cells in blind rats. J Neurosci, 18: 1818-1826

30 SAVE E, POUCET B (2000) Hippocampal-parietal cortical interactions in spatial cognition. Hippocampus, 10: 491-494

31 SAVE E, POUCET B, FOREMAN N, BUHOT MC (1992) Object exploration and reactions to spatial and nonspatial changes in hooded rats following damage to parietal cortex or hippocampal formation. Behav Neurosci, 106: 447-456

32 SEAMANS J, PHILLIPS A (1994) Selective Memory Impairments produced by transient lidocaine-induced Lesion of Nucleus Accumbens in Rats. Behav Neurosci, 108: 456-468

33 TORREALBA F, OLAVARRÍA J, CARRASCO MA (1984) Cortical connections of the anteromedial extrastriate visual cortex in the rat. Exp Brain Res, 56: 543-549

34 VOGT BA, MILLER MW (1983) Cortical connections between rat cingulate cortex and visual, motor and postsubicular cortices. J Comp Neurol, 216: 192-210

35 WYSS JM, VANGROEN T (1992) Connections between the retrosplenial cortex and the hippocampal formation in the rat: a review. Hippocampus, 2: 1-11 
